\title{
MALDI-TOF MS Analysis of Soluble PEG Based Multi-Step Synthetic Reaction Mixtures with Automated Detection of Reaction Failure
}

\author{
Christine Enjalbal, Patrice Ribière, Frédéric Lamaty, \\ Neerja Yadav-Bhatnagar, Jean Martinez, and Jean-Louis Aubagnac \\ Laboratoire des Aminoacides Peptides et Protéines, Universités Montpellier I \& II, Montpellier, France
}

Macromolecules of tunable solubility, used to mimic inert insoluble materials while maintaining solution conditions, allowed the performance of efficient supported organic chemistry and facilitated in situ reaction monitoring. To satisfy the high throughput requirements of automated synthetic processes, organic syntheses carried out on bifunctional polyethylene glycol polymers $\left(\mathrm{PEG}_{3400}-\mathrm{OH}\right)$ were monitored step-by-step by matrix assisted laser desorption ionization-time of flight mass spectrometry (MALDI-TOF MS). A protocol was designed to control the ionization mechanism of such polymers exhibiting high affinity for alkali metal cations. Automated, rapid, and reliable data interpretation was performed by an in-house developed visual basic application relying on the sodiated ion accurate monoisotopic mass measurement. The methodology was illustrated through the monitoring of a six-step synthetic scheme. (J Am Soc Mass Spectrom 2005, 16, 670-678) (c) 2005 American Society for Mass Spectrometry

W ater-soluble polyethylene glycol polymers (PEG) [1], often used as biomaterials for drug delivery [2], were also investigated as an inert material to perform supported organic reactions [3-5]. Since such polymer solubility/insolubility is simply tuned by choosing an appropriate solvent, these macromolecules present a viable alternative to solid-phase strategies for performing multistep parallel syntheses $[3,4]$. When dissolved in water or in organic solvents, PEG is subjected to reactions which are conducted in homogeneous media, alleviating the reactivity discrepancy sometimes observed under heterogeneous solidphase synthesis conditions. Otherwise, when precipitated, PEG mimics insoluble polymeric materials and can be handled as a solid-phase resin. In particular, precipitation of the polymer allows straightforward purification by filtration, excess reagents being drained as in the standard solid-phase strategy. Soluble polymer supported syntheses thus combine advantages of both solution-phase and solid-phase methodologies [6,7]. Such so-called liquid-phase reactions that can be simply described as an end group functional modification of the polymer were investigated for preparing organic molecules $[8,9]$ as well as combinatorial libraries $[4$, $10-13]$.

Published online March 11, 2005

Address reprint requests to Dr. C. Enjalbal, UMR 5810, LAPP, Université Montpellier II, 34095 Montpellier Cedex 5, France. E-mail: enjalbal@univmontp2.fr

* Also with Aventis Pharma, 102 route de Noisy, 93325 Romainville Cedex, France.
From an analytical point of view, PEG samples are suitable for conventional spectroscopic methods such as nuclear magnetic resonance (NMR) [14] and mass spectrometry (electrospray ionization \{ESI\} $[15,16]$ and matrix assisted laser desorption \{MALD\} [17-19]) which is not the case for resins. Organic molecules attached to such insoluble materials are cleaved from the support and released in solution for characterization (cleave and analyze strategy) [20]. Alternatively, direct in situ identification of anchored compounds is feasible provided that specific analytical techniques are available, such as cross polarization-magic angle spinning (CP-MAS) NMR [21-23] or static-secondary ion mass spectrometry (S-SIMS) [24-27]. In contrast to solid-phase synthesis, reactions carried out on soluble polymers were directly monitored by simply dissolving the macromolecules in an appropriate solvent. No release in solution of the growing molecule from the polymer backbone was thus required, characterization of the end group structure of the substituted PEG sample being sufficient to assess reaction output. Besides, such nondestructive analysis is particularly suited for multistep syntheses. The fact that the analyses can be performed on any standard laboratory equipment (NMR and MALDI or ESI MS) constituted a supplementary reason to prefer soluble polymer syntheses methodology as an alternative to the solid-phase strategy.

We report in this paper a simple automated analytical control of PEG 3400-mediated syntheses based on a MALDI MS instrument (Ultraflex, Bruker Daltonics, Wissembourg, France) to acquire positive or negative ion mass spectra that were then interpreted by an 


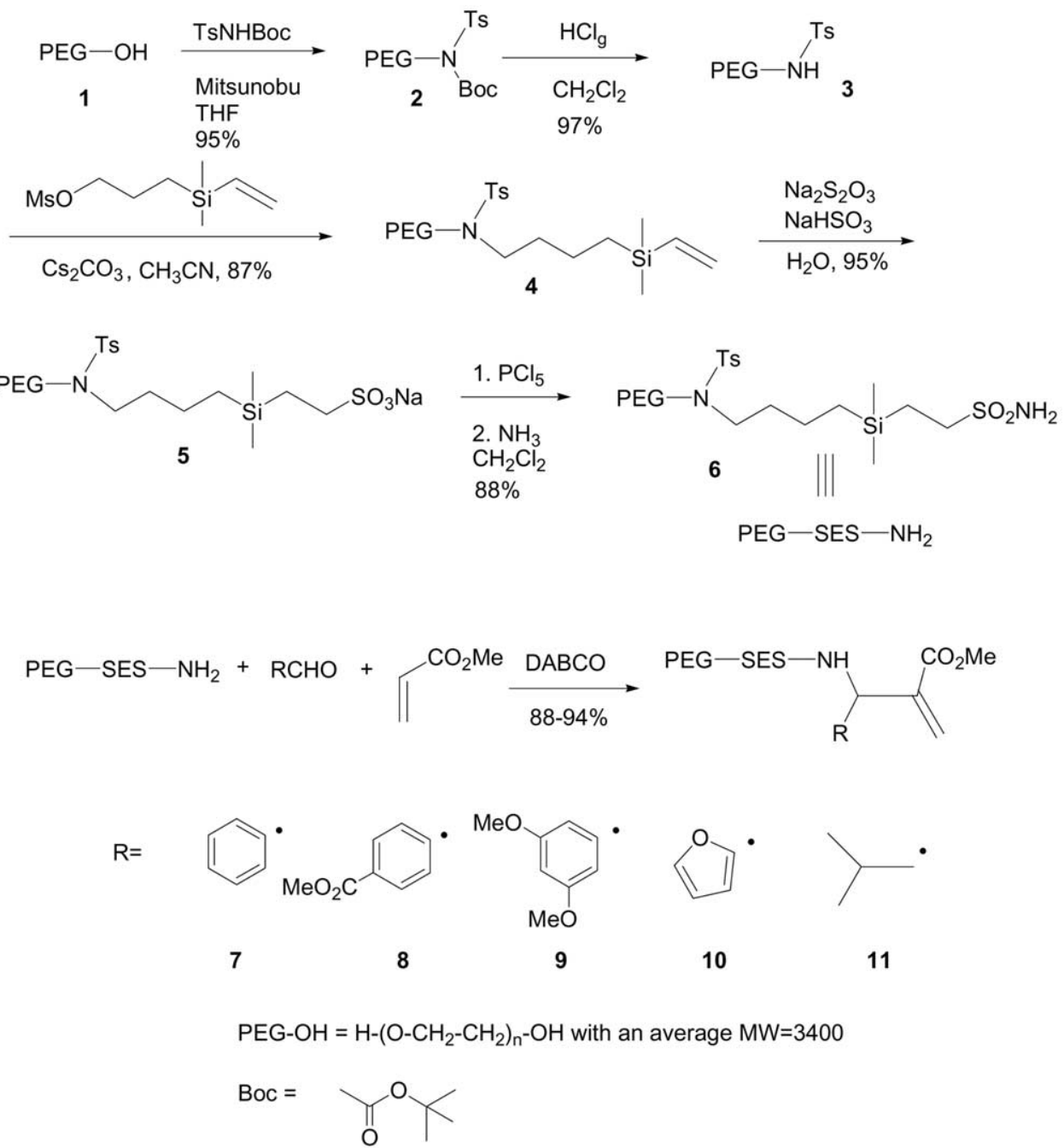

Scheme 1. Six-stage synthesis followed step-by-step by MALDI-TOF mass spectrometry.

in-house developed visual basic application. Although ESI MS coupled to liquid chromatography (LC/ESI-MS) allowed the unraveling of mixtures [16], the choice of MALDI MS was governed by the fact that far simpler mass spectra were generated, thus allowing easier and more rapid data interpretation. Such automated high throughput process, including data acquisition and interpretation, could be applied to the monitoring of multistep synthesis or to library profiling in combinatorial chemistry.

\section{Experimental}

\section{Syntheses}

The synthesis which was analyzed step-by-step by MALDI-TOF MS analysis is described in Scheme 1. A new sulfonyl linker of the SES-type ( trimethylsilylethyl sulfonyl) has been prepared and used in the preparation of unsaturated $\beta$-aminoesters by an aza-BaylisHillman reaction [9].

Bifunctional PEG 1 with an average molecular weight of 3400 was transformed in the tosyl amino PEG 3 by successive Mitsunobu reaction with TsNHBoc and acidic hydrolysis of the Boc group. Alkylation of $\mathbf{3}$ with the mesylate of 3-(dimethylvinyl)silylpropanol in the presence of $\mathrm{Cs}_{2} \mathrm{CO}_{3}$ provided 4 . Sulfonate 5 was obtained by reaction of 4 with $\mathrm{Na}_{2} \mathrm{~S}_{2} \mathrm{O}_{3}$. Action of $\mathrm{PCl}_{5}$ on 5 yielded the corresponding sulfonyl chloride which was transformed in 6 by reaction with ammonia. 6 participated in aza-Baylis-Hillman reactions with methyl acrylate and with various aromatic aldehydes to provide the PEG-supported $\beta$-aminoesters 7-11.

\section{Mass Spectrometry}

The PEG samples were dissolved in a $10 \mathrm{mg} / \mathrm{ml}$ THF: 18.2 M $\Omega . c m$ (MilliQ grade) water (90:10 vol:vol) solution. Dithranol $(20 \mathrm{mg} / \mathrm{ml}$ in THF) was used as a matrix. A $10 \mathrm{mg} / \mathrm{ml}$ solution of sodium trifluoroacetate in Milli-Q grade water was used as a cationizing agent. Fifteen $\mu \mathrm{l}$ of the matrix solution were mixed with $5 \mu \mathrm{l}$ of the PEG solution and $0.5 \mu \mathrm{l}$ of the cationization solution 


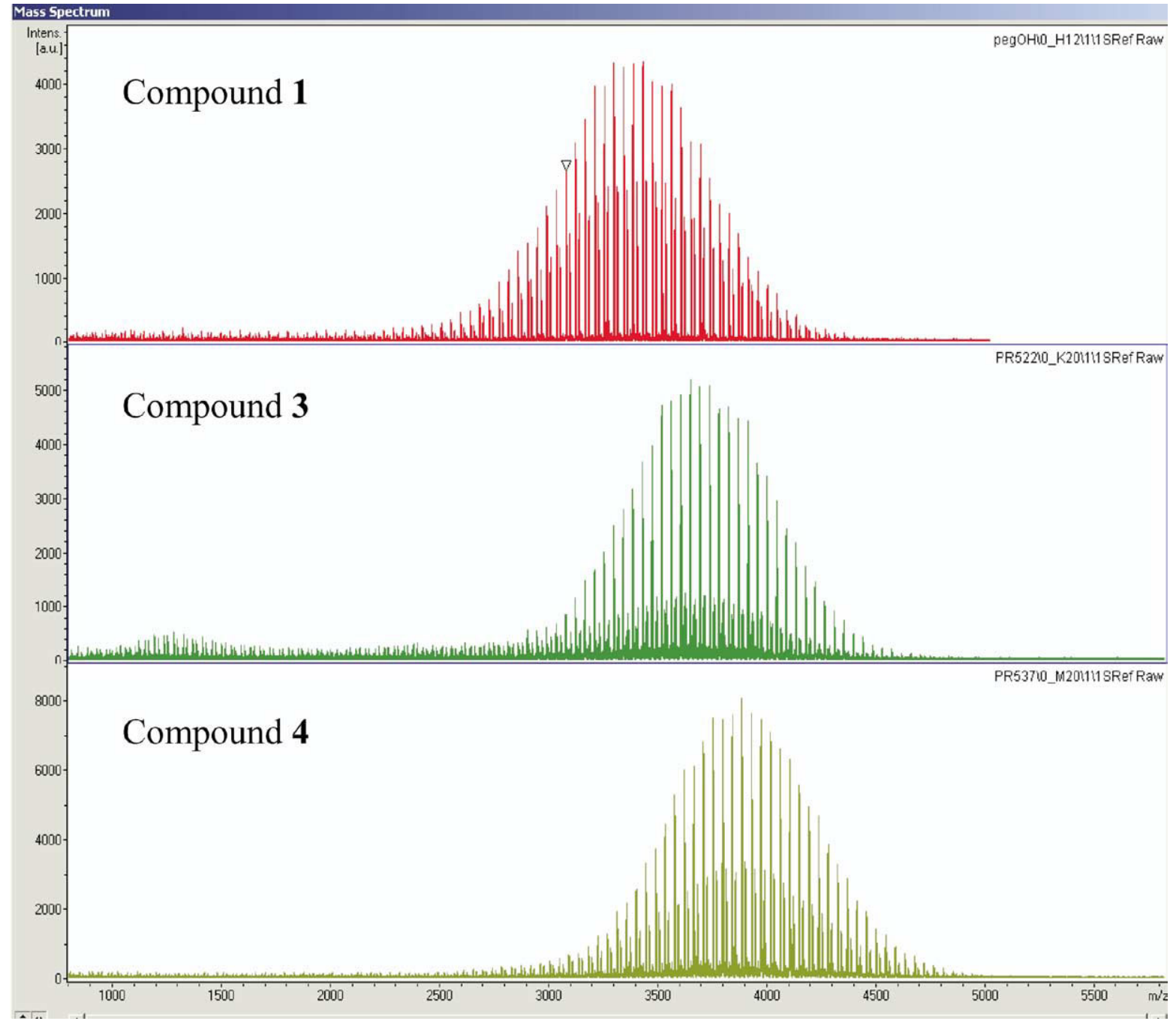

Figure 1. MALDI mass spectra of Compounds 1, 3, and 4 described in Scheme 1.

in a $1.5 \mathrm{ml}$ original Eppendorf tube (Eppendorf AG, Hamburg, Germany). MALDI-TOF mass spectra were acquired using the reflectron mode on an Ultraflex TOF-TOF mass spectrometer (Bruker Daltonik GmbH, Bremen, Germany). The serial instrument was equipped with a $337 \mathrm{~nm}, 50 \mathrm{~Hz} \mathrm{~N}$ Laser used with a $33 \%$ attenuation level. The acceleration voltage was set to $19 \mathrm{kV}$, the pulsed extraction voltage to $17.15 \mathrm{kV}$, the extraction delay to $280 \mathrm{~ns}$, and the reflector voltage to 20 $\mathrm{kV}$. The achieved peak resolution was comprised between 10,000 and 30,000, depending on the peaks.

External calibrations were performed with PEG 4000 (Fluka Chemie GmbH, Buchs, Switzerland). One calibrant spot was disposed in the center of a 9-sample square and used to calibrate these samples (the overall distance between the calibration spot and the measured sample was always between 4 and $7 \mathrm{~mm}$ ). This procedure ensures reaching mass errors far below the 40ppm-level. On an externally calibrated PEG-OH sample, all the measured peaks (42) showed errors below the 40 ppm level, more than $97 \%$ being below 30 ppm,
$83 \%$ below 20 ppm, and 65\% below 5 ppm. Restricting the measure to the calibrant-defined mass-range $(\mathrm{m} / \mathrm{z}$ $3000-4000)$, more than $95 \%$ of the peaks were measured with errors below 5 ppm.

For all studied compounds, the most abundant distribution was related to sodiated species whereas the less intense signals were attributed to potassium adducts (Figure 1, Compound 1). So, only two data remained unknown when studying any of the most abundant ions: the number of ethylene oxide units (n) and the structure of the end group $R$.

\section{Data Mining}

The algorithm is given in Scheme 2.

\section{Results and Discussion}

\section{Analytical Strategy}

Even though the analysis of the mixtures was quite difficult and the matrix needed to be carefully chosen 


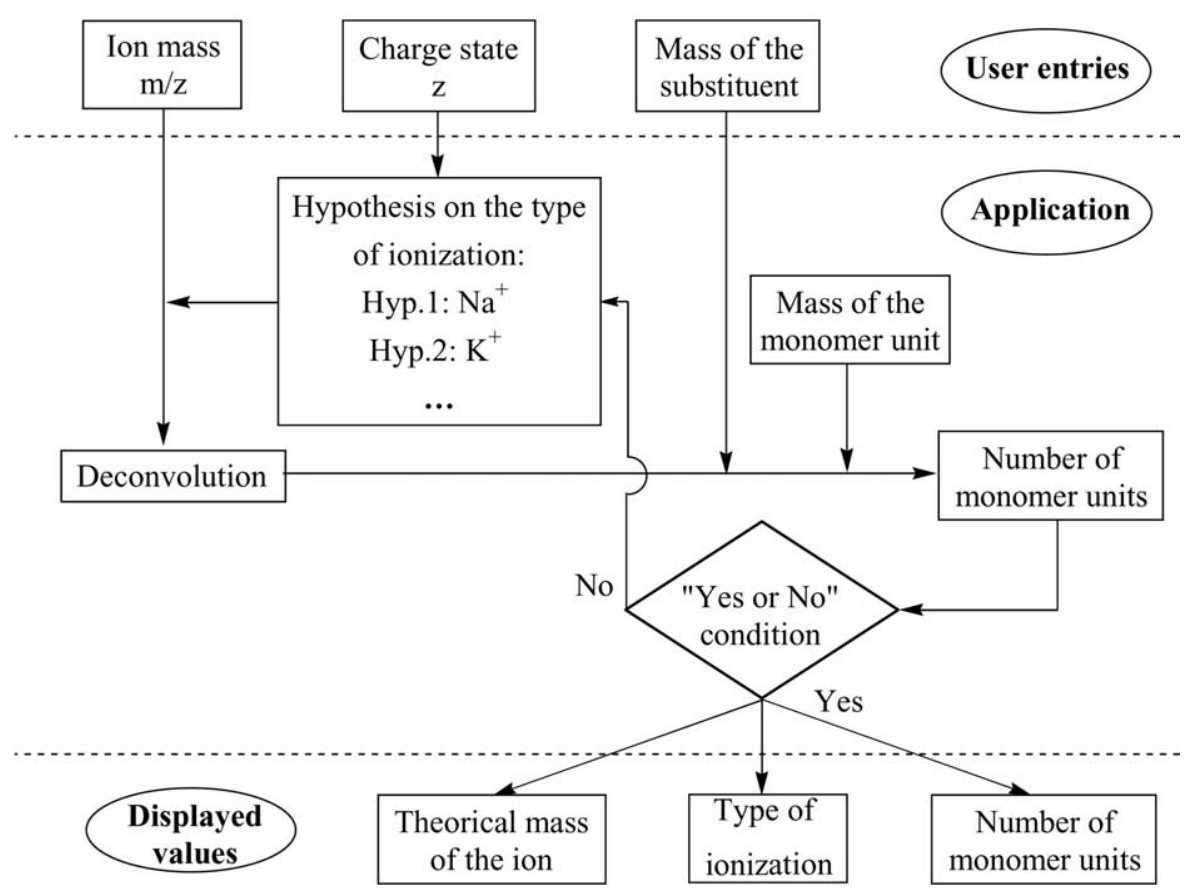

Scheme 2. Data mining algorithm.

[18], MALDI was preferred over ESI to conduct high throughput analyses provided that an appropriate protocol was available to guarantee efficient PEG detection for all studied samples. The six-step synthesis described in Scheme 1 was followed step-by-step. Addition of sodium trifluoroacetate during the MALDI matrix solution preparation was set up to provide an efficient ionization by cationization with sodium of the PEG samples. Residual cationization with potassium was nevertheless observed because of the high affinity of PEG for both sodium and potassium [28], giving rise to a second Gaussian distribution. Although the extent of cationization with potassium was found to vary sample-to-sample (for instance, as shown in Figure 1, Compounds 1 and 4 exhibited much more intense (MK) ${ }^{+}$ signals compared with Compound 3). Such parasite $(\mathrm{MK})^{+}$ions always remained less abundant than the corresponding sodiated species $(\mathrm{MNa})^{+}$.

For the commercially available polymer selected for the syntheses (bifunctional $\mathrm{PEG}_{3400}-\mathrm{OH}$ ), the number of ethylene oxide units (n) varied roughly from 60 to 100 in the oligomeric distribution centered around $3400 \mathrm{Da}$. Starting from native PEG (PEG-OH) which was subjected to successive chemical reactions to provide the expected macromolecules (PEG-R), the intended end group modification $(\mathrm{OH} \rightarrow \mathrm{R})$ was validated upon MALDI analysis. For any detected ion in the mass spectra, three unknown data needed to be elucidated simultaneously. First, the nature of the charge agent (X $=\mathrm{Na}$ or $\mathrm{K}$ ) must be known to identify the ion type and thus interpret correctly the mass data. Second, the knowledge of the number of ethylene oxide units (n) allowed identifying the oligomer under study, and third, the structure of the end group (R) enabled characterizing the chemical structure.

The previously reported methods to control supported syntheses carried out on PEG by MALDI MS [17] implied direct comparison of the product and native PEG mass spectra. A mass increment was calculated between the starting material and the product. An ion was selected in the mass spectrum of PEG-OH, the mass increment was added and, provided that the corresponding value was detected in the product mass spectrum, the reaction was validated. This methodology works well as long as the following criterion is satisfied: the ionization result (protonated or cationized species) must be identical for the two samples. We have developed a more general method which is based on the knowledge of the ionization output allowing direct mass spectral interpretation instead of relative mass increment measurement between two shifted ion distributions of a reference and the product under study. Indeed, the width of the recorded PEG 3400 Gaussian distributions was spread over several ions (approximately 20 signals of fair abundance, all separated by 44 $\mathrm{Da}$, corresponding to a mass range of $880 \mathrm{Da}$ ). Small differences between starting material and product masses could thus be difficult to spot, being included within the Gaussian distribution.

\section{Data Mining}

The high resolution of the TOF analyzer used in this study allowed recording of the isotopic cluster for each ion of the oligomeric distribution (Figure 2). As stated in the Experimental section, external calibration en- 
Compound 1

\begin{tabular}{|c|c|c|c|c|}
\hline $\begin{array}{c}\mathbf{m} / \mathbf{z} \\
(\mathbf{M}+\mathbf{N a})^{+}\end{array}$ & $\begin{array}{c}\text { Theoretical } \\
\text { mass }\end{array}$ & $\begin{array}{c}\text { Mass } \\
\text { accuracy } \\
\text { (ppm) }\end{array}$ & $\begin{array}{c}\text { Calculated } \\
\mathbf{n}\end{array}$ & $\begin{array}{c}\text { «Error » } \\
\text { (pass /fail } \\
\text { threshold } \\
\text { set at 5 10-3) }\end{array}$ \\
\hline 2990.744 & 2990.756 & 4 & 66.9997 & $0.310^{-3}$ \\
3034.776 & 3034.782 & 2 & 67.9999 & $0.110^{-3}$ \\
3078.798 & 3078.808 & 3 & 68.9998 & $0.210^{-3}$ \\
3122.824 & 3122.834 & 3 & 69.9998 & $0.210^{-3}$ \\
3166.850 & 3166.861 & 4 & 70.9998 & $0.210^{-3}$ \\
3210.878 & 3210.887 & 3 & 71.9998 & $0.210^{-3}$ \\
3254.909 & 3254.913 & 1 & 72.9999 & $0.110^{-3}$ \\
3298.933 & 3298.939 & 2 & 73.9999 & $0.110^{-3}$ \\
3342.965 & 3342.965 & 0 & 75.0000 & $0.0110^{-3}$ \\
3386.987 & 3386.992 & 2 & 75.9999 & $0.110^{-3}$ \\
3431.012 & 3431.018 & 2 & 76.9998 & $0.110^{-3}$ \\
\hline
\end{tabular}

Isotopic cluster of $\mathrm{m} / \mathrm{z} 2990.744$

Compound 3

\begin{tabular}{|c|c|c|c|c|}
\hline $\begin{array}{c}\mathbf{m} / \mathbf{z} \\
(\mathbf{M + N a})^{+}\end{array}$ & $\begin{array}{c}\text { Theoretical } \\
\text { mass }\end{array}$ & $\begin{array}{c}\text { Mass } \\
\text { accuracy } \\
\text { (ppm) }\end{array}$ & $\begin{array}{c}\text { Calculated } \\
\mathbf{n}\end{array}$ & $\begin{array}{c}\text { « Error » } \\
\text { (pass /fail } \\
\text { threshold } \\
\text { set at 5 10-3) }\end{array}$ \\
\hline 3164.846 & 3164.724 & 38 & 64.0027 & $2.710^{-3}$ \\
3208.870 & 3208.753 & 36 & 65.0026 & $2.610^{-3}$ \\
3252.897 & 3252.779 & 36 & 66.0027 & $2.710^{-3}$ \\
3296.929 & 3296.806 & 37 & 67.0028 & $2.810^{-3}$ \\
3340.955 & 3340.832 & 37 & 68.0028 & $2.810^{-3}$ \\
3384.979 & 3384.858 & 36 & 69.0027 & $2.710^{-3}$ \\
3429.010 & 3428.884 & 37 & 70.0028 & $2.810^{-3}$ \\
3473.032 & 3472.910 & 35 & 71.0028 & $2.810^{-3}$ \\
3517.062 & 3516.937 & 36 & 72.0028 & $2.810^{-3}$ \\
3561.087 & 3560.963 & 35 & 73.0028 & $2.810^{-3}$ \\
3605.104 & 3604.989 & 32 & 74.0026 & $2.610^{-3}$ \\
\hline
\end{tabular}

Isotopic cluster of $\mathrm{m} / \mathrm{z} 3605.104$

Compound 4

\begin{tabular}{|c|c|c|c|c|}
\hline $\begin{array}{c}\mathbf{m} / \mathbf{z} \\
(\mathbf{M}+\mathbf{N a})^{+}\end{array}$ & $\begin{array}{c}\text { Theoretical } \\
\text { mass }\end{array}$ & $\begin{array}{c}\text { Mass } \\
\text { accuracy } \\
\text { (ppm) }\end{array}$ & $\begin{array}{c}\text { Calculated } \\
\mathbf{n}\end{array}$ & $\begin{array}{c}\text { « Error » } \\
\text { (pass /fail } \\
\text { threshold } \\
\text { set at 5 10 10 }\end{array}$ \\
\hline 3621.036 & 3621.036 & 0 & 68.0000 & $210^{-6}$ \\
3665.065 & 3665.062 & 1 & 69.0001 & $610^{-5}$ \\
3709.092 & 3709.088 & 1 & 70.0001 & $810^{-5}$ \\
3753.120 & 3753.115 & 1 & 71.0001 & $110^{-4}$ \\
3797.146 & 3797.141 & 1 & 72.0001 & $110^{-4}$ \\
3841.172 & 3841.167 & 1 & 73.0001 & $110^{-4}$ \\
3885.200 & 3885.193 & 2 & 74.0002 & $210^{-4}$ \\
3929.225 & 3929.219 & 2 & 75.0001 & $110^{-4}$ \\
\hline
\end{tabular}

Isotopic cluster of $\mathrm{m} / \mathrm{z} 3885.200$

Figure 2. MALDI mass spectra of Compounds 1,3, and 4: Accurate mass measurement of the most abundant ions with the display of one isotopic cluster. 


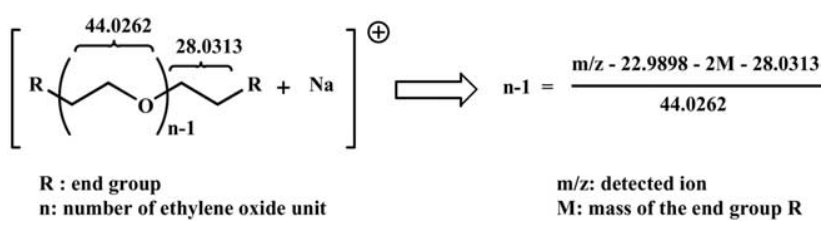

Scheme 3. Equation to calculate the number of ethylene oxide units that compose any detected ion in MALDI-TOF mass spectra.

sured mass measurement errors far below the $40 \mathrm{ppm}$ level. Such accuracy allowed us to design specific software (visual basic application) [16] that relies only on this value to validate or not to validate the output of the reaction. The mass of the expected end group was entered in the software and the number of ethylene oxide units (n) was calculated within seconds from the formula given in Scheme 3. As long as a very accurate mass measurement was available for $\mathrm{m} / \mathrm{z}$, the recovered value of $\mathrm{n}$ had to be very close to an integer. The deviation from the theoretical value, termed "error", was considered as an acceptance criterion providing a pass/fail response, commonly used to profile combinatorial libraries [29-31]. The aim of the designed data management protocol was to rapidly spot problematic syntheses. Since the algorithm was based on the knowledge of the end group $(\mathrm{R})$ to calculate the EO number (n), no information on the nature of the analyzed products was available when R was not correct. Only manual inspection of the recorded mass spectra could unravel the unknown structures.

The validity of such automated high throughput data mining relied on the chosen threshold ("error $=5$ $\times 10^{-3 \prime \prime}$ ) to distinguish between successful and failed reactions. A very small "error" was tolerated to confirm the structure of the synthesized product as illustrated in Table 1 and Figure 2. Any value within this "error" meant that the calculated mass of the PEG substituents was correct and thus that the expected compounds have been synthesized.

Other polymeric supports used to perform organic syntheses [5] could be subjected to the described process provided that the equation of Scheme 3 was modified by replacing the mass of an ethylene oxide unit by the mass of the new monomer. One could also envisage leaving the mass of the monomer as a datum specified by the user in order to cover all situations encountered in soluble polymer supported synthesis.

\section{Application}

The multistep synthesis described in Scheme $\mathbf{1}$ was subjected to MALDI-TOF MS. To illustrate the results, the MALDI mass spectra of Compounds 1, 3, and 4 were selected (Figures 1 and 2). Narrowly dispersed mass distributions $(\mathrm{MNa}+/ \mathrm{MK}+$ ions) were observed. In some cases, the recorded mass spectra were also displaying low molecular weight product ions appearing outside the expected polymer distribution as shown in Figure 3 (Compound 10, expected $\mathrm{MNa}+$ ions noted by closed circles). Two explanations can be envisaged to account for such ions. First, truncated polymeric chains could be produced either during MALDI analysis by in-source fragmentations or during sample work-up (inappropriate conditions during synthesis, purification, or storage). Second, incomplete reactions are common in chemistry leading to the presence of sideproducts (unreacted starting materials, eventually monosubtituted PEG, or unexpected end group modification). These products could be stable in the remaining synthetic steps or react further, providing more and more complex mixtures with ions detected over a large mass range. Even in such a situation (Compound 10, Figure 3), the recorded mass spectrum allowed visualization of the expected polymer distribution and was suitable for automated data mining. Otherwise, further analyses such as MS/MS and LC/ESI-MS experiments are required.

All compounds, except Compound 2, showed an "error" less than $5 \times 10^{-3}$ (Table 1 and Figure 2) and were thus validated. For the analysis that was out of

Table 1. Calculated "error" for high throughput MALDI-TOF data mining for Compounds $\mathbf{1}$ to $\mathbf{1 1}$

\begin{tabular}{|c|c|c|c|c|c|}
\hline $\mathrm{N}^{\circ}$ & $\mathrm{R}$ & $2 \mathrm{M}$ & $\begin{array}{l}\text { Most abundant } \\
\text { observed ion }\end{array}$ & $\mathrm{n}$ & $\begin{array}{c}\text { Error* } \\
\text { (threshold } \\
\text { set at } 510^{-3} \text { ) }\end{array}$ \\
\hline 1 & $\mathrm{OH}$ & 34.0055 & 3386.987 & 75.9999 & $0.110^{-3}$ \\
\hline 2 & $\mathrm{C}_{12} \mathrm{H}_{16} \mathrm{NO}_{4} \mathrm{~S}$ & 540.1600 & 3605.104 & 69.4575 & $45710^{-3}$ \\
\hline 3 & $\mathrm{C}_{7} \mathrm{H}_{8} \mathrm{NO}_{2} \mathrm{~S}$ & 340.0552 & 3605.104 & 74.0026 & $2.610^{-3}$ \\
\hline 4 & $\mathrm{C}_{15} \mathrm{H}_{24} \mathrm{NO}_{2} \mathrm{SSi}$ & 620.2594 & 3885.200 & 74.0002 & $0.210^{-3}$ \\
\hline 5 & $\mathrm{C}_{15} \mathrm{H}_{25} \mathrm{NO}_{5} \mathrm{~S}_{2} \mathrm{SiNa}$ & 828.1682 & 4092.1176 & 73.9776 & $2210^{-3}$ \\
\hline 6 & $\mathrm{C}_{15} \mathrm{H}_{27} \mathrm{~N}_{2} \mathrm{O}_{4} \mathrm{~S}_{2} \mathrm{Si}$ & 782.2363 & 4003.1927 & 73.0011 & $1.110^{-3}$ \\
\hline 7 & $\mathrm{C}_{26} \mathrm{H}_{37} \mathrm{~N}_{2} \mathrm{O}_{6} \mathrm{~S}_{2} \mathrm{Si}$ & 1130.3725 & 4263.1295 & 70.9978 & $2.210^{-3}$ \\
\hline 8 & $\mathrm{C}_{28} \mathrm{H}_{39} \mathrm{~N}_{2} \mathrm{O}_{8} \mathrm{~S}_{2} \mathrm{Si}$ & 1246.3834 & 4423.1880 & 71.9982 & $1.710^{-3}$ \\
\hline 9 & $\mathrm{C}_{28} \mathrm{H}_{41} \mathrm{~N}_{2} \mathrm{O}_{8} \mathrm{~S}_{2} \mathrm{Si}$ & 1250.4148 & 4383.3333 & 71.0014 & $1.410^{-3}$ \\
\hline 10 & $\mathrm{C}_{24} \mathrm{H}_{35} \mathrm{~N}_{2} \mathrm{O}_{7} \mathrm{~S}_{2} \mathrm{Si}$ & 1110.3310 & 4199.1453 & 69.9997 & $1.010^{-3}$ \\
\hline 11 & $\mathrm{C}_{24} \mathrm{H}_{41} \mathrm{~N}_{2} \mathrm{O}_{6} \mathrm{~S}_{2} \mathrm{Si}$ & 1090.4351 & 4267.1743 & 71.9967 & $3.210^{-3}$ \\
\hline
\end{tabular}

The discrepancy for Compound 5, which showed an error slightly superior to the acceptance threshold, may be due to its anionic nature (possibility of protonation or counterion exchange). MS analysis of the same sample in the negative mode was found more suitable.

*Error calculated as (theoretical value of $n-$ calculated value of $n$ ) 


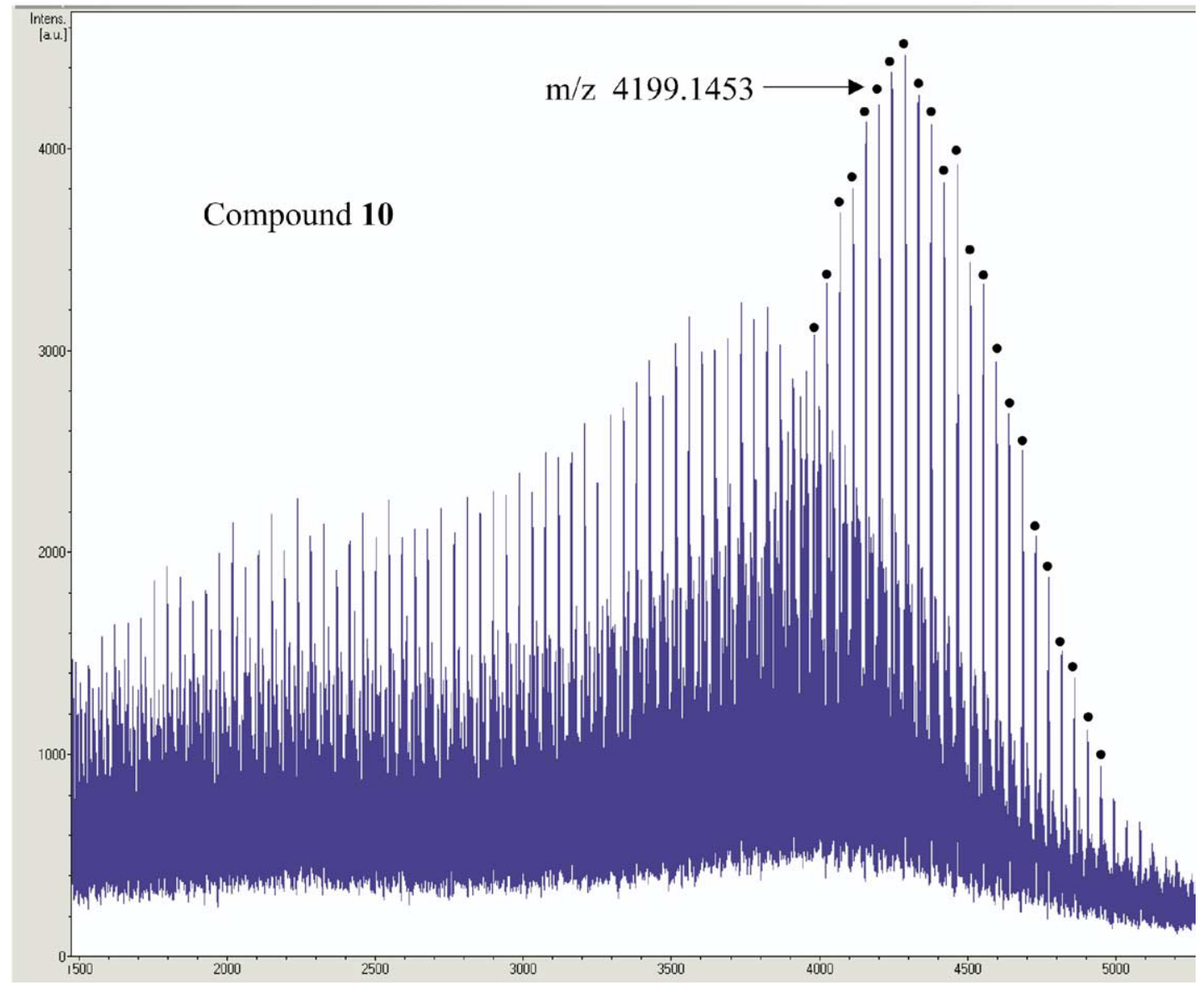

Figure 3. MALDI mass spectrum of Compound $\mathbf{1 0}$ described in Scheme $\mathbf{1}(\mathrm{MNa}+$ ions noted by closed circles).

range (Compound 2), the question of an analytical failure was raised before concluding that the targeted reaction had not occurred. Any reactivity occurring during the analysis because of the use of an inappropriate matrix would provide erroneous results. In fact, fragmentation of the acid-sensitive Boc group occurred readily during the MALDI-TOF analysis no matter what the nature of the acidic matrix used (dihydroxybenzoic acid, dithranol, cinnamic acid, and azathiothymic acid), giving Compound 2 the same MALDI mass spectrum as Compound 3. This problem was expected [18], since MALDI analysis of acid-sensitive molecules requires the use of nonacidic matrices, and Compound 2 identity was checked by both NMR and ESI MS using nonacidic solvents. In the case of multistep syntheses, such situation can be rapidly evidenced by considering the compound next in line in the synthesis. If this expected molecule has been prepared, the previous reaction was correct and the analysis was faulty. With regard to combinatorial library profiling, since each analysis is independent, such reasoning cannot be applied.

If we consider Compound 2, the calculation described in Scheme 3 gave a number of ethylene oxide units different from an integer (70.4590 for the ion at $\mathrm{m} / \mathrm{z}$
3649.2). Obviously, this sample required further investigation. Despite the addition of sodium trifluoroacetate, one can argue that ionization occurred preferentially by cationization with potassium. Such a hypothesis was even more pertinent if the ionization mechanism was not controlled. According to the calculation described in Scheme 3, the number of ethylene oxide units was recovered by changing the mass of sodium $(22.9898 \mathrm{Da})$ with the mass of potassium (38.9637). A value of 70.0962 was obtained for Compound 2. Although these figures did not differ much from an integer, the corresponding "error" $\left(96 \times 10^{-3}\right)$ was far off the limit of $5 \times 10^{-3}$ deduced from the mass spectrometer performance. The drastic threshold of $5 \times$ $10^{-3}$ allowed dismissing this mass spectrum that could otherwise have been accounted for by direct mass increment measurement for the expected compound (in the form of potassium adducts). However, our goal was to apply the same protocol to all studied samples in order to achieve an automated high throughput process. Although the most abundant ions were always related to sodiated species, the interpretation software was implemented with a second calculation based on cationization with potassium that could be triggered in the case of rejected mass spectra. 


\section{What About a Low Performance Mass Spectrometer?}

One can distinguish two situations: either low mass measurement accuracy or low resolution. In the first case (a high resolution mass spectrometer runs with a calibration protocol giving at best a mass measurement of $40 \mathrm{ppm}$ ), the acceptance threshold ("error") was chosen according to the following consideration. Since 1 Da represents the smallest mass difference between two consecutive reactions, two bifunctional PEG compounds would be separated by at least $2 \mathrm{Da}$. One should be able to distinguish two isotopic clusters differing by $2 \mathrm{Da}$ and centered around $3400 \mathrm{Da}$ or more. The acceptance threshold was thus chosen accordingly: the minimum "error" on the number of ethylene oxide unit calculation equaled 2 Da divided by the monoisotopic mass of the ethylene oxide monomer $(2 / 44.0262=$ 0.04542 ). An approximated value of $5 \times 10^{-2}$ was implemented in the data interpretation software. In the second case, when the resolution power of the mass spectrometer did not allow the required isotopic mass measurement, knowledge of the average mass would be roughly $2 \mathrm{Da}$ above the monoisotopic value since an increase of $1 \mathrm{Da}$ is estimated every 1500 mass units [32, 33] (for instance, the most abundant ion in the mass spectrum of Compound $\mathbf{1}$ corresponds to the molecular formula $\mathrm{C}_{150} \mathrm{H}_{302} \mathrm{O}_{76}$ : monoisotopic mass of 3079.9767 and average mass of 3081.7823 giving a mass difference of $1.8 \mathrm{Da}$ ). The same calculation presented in Scheme 3 could be performed by considering average masses instead of monoisotopic values. A correct data assignment would have been obtained as long as the acceptance criterion was kept as low as possible.

\section{Conclusions}

Rapid MALDI-TOF mass spectra acquisition and subsequent automated spectral data interpretation ensured efficient reaction monitoring at every stage of multistep synthetic schemes. Compound identification was straightforward except for acid-sensitive molecules that were found to react during the analysis. The analysis protocol provided high throughput macromolecules characterization and was thus particularly suited to profile combinatorial PEG-based libraries. Compounds that failed to be analyzed or reactions that led to unexpected products could be reanalyzed by ESI-MS or, in the case of mixtures, by LC/ESI-MS. Such an analytical process is similar to the one used in proteomics where high throughput peptide mass fingerprints are produced by MALDI MS to perform database searching. In the case of unmatched data, specific peptides are then characterized by LC/ESI-MS to gain more information [34].üThus,üanalysisüisüoptimizedübyürapidly validating successful syntheses and by spending most of the time on a few spotted problematic samples.

\section{Acknowledgments}

The authors thank P. Lefeuvre, P.-O. Schmit, and A. Asperger from Bruker Daltonics (Wissembourg, France) for conducting the MALDI-TOF MS analyses.

\section{References}

1. Harris, J. M.; Zalipsky, S. Poly(ethylene glycol): Chemistry and Biological Applications. ACS Symposium Series 680: Washington, DC, 1997.

2. Greenwald, R. B.; Choe, Y. H.; McGuire, J.; Conover, C. D. Effective drug delivery by PEGylated drug conjugates. Adv. Drug Delivery Rev. 2003, 55, 217-250.

3. Bayer, E.; Mutter M. Liquid phase synthesis of peptides. Nature 1972, 237, 512-513.

4. Hans, H.; Wolfe, M. M.; Brenner, S.; Janda, K. D. Liquid-phase combinatorial synthesis. Proc. Natl. Acad. Sci. U.S.A. 1995, 92, $6419-6423$

5. Gravert, D. J.; Janda, K. D. Synthesis on soluble polymers: New reactions and the contruction of small molecules. Curr. Opin. Chem. Biol. 1997, 1, 107-113.

6. Wentworth, P. Recent developments and applications of liquid-phase strategies in organic synthesis. Trends Biotechnol. 1999, 17, 448-452.

7. Gravert, D. J.; Janda, K. D. Organic synthesis on soluble polymer supports: Liquid-phase methodologies. Chem. Rev. 1997, 33, 489-509.

8. Varray, S.; Gauzy, C.; Lamaty, F.; Lazaro, R.; Martinez, J. Synthesis of cyclic amino acid derivatives via ring closure metathesis on a poly(ethylene glycol) supported substrate. $J$. Org. Chem. 2000, 65, 6787-6790.

9. Ribière, P.; Enjalbal, C.; Aubagnac, J.-L.; Yadav-Bhatnagar, N.; Martinez, J.; Lamaty, F. Preparation of poly(ethylene glycol) sulfonamide: Synthesis of $\mathrm{N}$-supported $\beta$-aminoesters via the Aza-Baylis-Hillman reaction. J. Comb. Chem. 2004, 6, 464-467.

10. Sun, C. M. Recent advances in liquid-phase combinatorial chemistry. Comb. Chem. High Throughput Screen. 1999, 2, 299-318.

11. Pan, P.-C.; Sun, C.-M. High-throughput combinatorial synthesis of substituted benzimidazolones. Tetrahedron Lett. 1999, 40, 6443-6446.

12. Sauvagnat, B.; Kulig, K.; Lamaty, F.; Lazaro, R.; Martinez, J. Soluble polymer-supported synthesis of $\alpha$-amino acid derivatives. J. Comb. Chem. 2000, 2, 134-142.

13. Sauvagnat, B.; Lamaty, F.; Lazaro, R.; Martinez, J. Poly(ethylene glycol) as solvent and polymer support in the microwave assisted parallel synthesis of amino acid derivatives. Tetrahedron Lett. 2000, 41, 6371-6375.

14. Shey, J.-Y.; Sun, C.-M. Liquid-phase combinatorial reaction monitoring by conventional ${ }^{1} \mathrm{H}$ NMR spectroscopy. Tetrahedron Lett. 2002, 43, 1725-1729.

15. Sauvagnat, B.; Enjalbal, C.; Lamaty, F.; Lazaro, R.; Martinez, J.; Aubagnac, J.-L. Step-by-step monitoring of a liquid phase organic synthesis by electrospray mass spectrometry. Rapid Commun. Mass Spectrom. 1998, 12, 1034-1037.

16. Enjalbal, C.; Lamaty, F.; Sanchez, P.; Suberchicot, E.; Ribière, P.; Varray, S.; Lazaro, R.; Yadav-Bhatnagar, N.; Martinez, J.; Aubagnac, J.-L. Characterization of soluble polymer supported organic compounds by LC/Electrospray ionization MS: Towards a complete automation of the liquid-phase process in combinatorial chemistry. Anal. Chem. 2003, 75, 175-184.

17. Thürmer, R.; Meisenbach, M.; Echner, H.; Weiler, A.; AlQawasmeh, R. A.; Voelter, W.; Korff, U.; Schmitt-Sody, W. Monitoring of liquid-phase peptide synthesis on soluble polymer supports via matrix-assisted laser desorption/ionization 
time-of-flight mass spectrometry. Rapid Commun. Mass Spectrom. 1998, 12, 398-402.

18. Enjalbal, C.; Sauvagnat, B.; Lamaty, F.; Lazaro, R.; Martinez, J.; Mouchet, P.; Roux, F.; Aubagnac J.-L. Chemical reactivity in matrix-assisted laser desorption/ionization mass spectrometry. Rapid Commun. Mass Spectrom. 1999, 13, 1775-1781.

19. Na, D. H.; Youn, Y. S.; Lee, K. C. Optimization of the PEGylation process of a peptide by monitoring with matrixassisted laser desorption/ionization time-of-flight mass spectrometry. Rapid Commun. Mass Spectrom. 2003, 17, 2241-2244.

20. Enjalbal, C.; Martinez, J.; Aubagnac, J.-L. Mass spectrometry in combinatorial chemistry. Mass Spectrom. Rev. 2000, 19, 139-161.

21. Sarkar, S. K.; Garagipati, R. S.; Adams, J. L.; Keifer, P. A. An NMR method to identify nondestructively chemical compounds bound to a single solid-phase bead for combinatorial chemistry applications. J. Am. Chem. Soc. 1996, 118, 2305-2306.

22. Chin, J.; Fell, B.; Shapiro, M. J.; Tomesch, J.; Wareing, J. R.; Bray, A. M. Magic angle spinning NMR for reaction monitoring and structure determination of molecules attached to Multipin crowns. J. Org. Chem. 1997, 62, 538-539.

23. Shapiro, M. J.; Wareing, J. R. NMR methods in combinatorial chemistry. Curr. Opin. Chem. Biol. 1998, 2, 372-375.

24. Brummel, C. L.; Vickerman, J. C.; Carr, S. A.; Hemling, M. E.; Roberts, G. D.; Johnson, W.; Weinstock, J.; Gaitanopoulos, D.; Benkovic, S. J.; Winograd, N. Evaluation of mass spectrometric methods applicable to the direct analysis of nonpeptide beadbound combinatorial libraries. Anal. Chem. 1996, 68, 237-242.

25. Carrasco, M. R.; Fitzgerald, M. C.; Oda, Y.; Kent, S. B. H. Direct monitoring of organic reactions on polymeric supports. Tetrahedron Lett. 1997, 38, 6331-6334.

26. Aubagnac, J.-L.; Enjalbal, C.; Subra, G.; Combarieu, R.; Bray, A. M.; Martinez, J. Application of time-of-flight secondary ion mass spectrometry to in situ monitoring of solid phase peptide synthesis on the Multipin system. J. Mass Spectrom. 1998, 33, 1094-1103.

27. Enjalbal, C.; Maux, D.; Combarieu, R.; Martinez, J.; Aubagnac, J.-L. Imaging combinatorial libraries by mass spectrometry: Peptide to organic-supported syntheses. J. Comb. Chem. 2003, 5, 102-109.

28. Bogan, M. J.; Agnes, G. R. Poly(ethylene glycol) doubly and singly cationized by different alkali metal ions: Relative cation affinities and cation-dependent resolution in a quadrupole ion trap mass spectrometer. J. Am. Soc. Mass Spectrom. 2002, 13, 177-186.

29. Shah, N.; Gao, M.; Tsutsui, K.; Lu, A.; Davis, J.; Scheuerman, R.; Fitch, W. L.; Wilgus, R. L. A novel approach to highthroughput quality control of parallel synthesis libraries. J. Comb. Chem. 2000, 2, 453-460.

30. Richmond, R.; Görlach; E.; Seifert, J.-M. High-throughput flow injection analysis-mass spectrometry with networked delivery of color rendered results: The characterization of liquid chromatography fractions. J. Chromatogr. A 1999, 835, 29-39.

31. Mallis, L. M.; Sarkahian, A. N.; Kulishoff, J. M.; Watts, W. L. Open-access liquid chromatophy/mass spectrometry in a drug discovery environment. J. Mass Spectrom. 2002, 37, 889896.

32. De Hoffmann, E.; Charette, J.; Stroobant, V. In Spectrométrie de masse, 2nd ed. Dunod: Paris, France, 1994, p 213.

33. Yergey, J.; Heller, D.; Hansen, G.; Cotter, R. J.; Fenselau, C. Isotopic distributions in mass spectra of large molecules. Anal. Chem. 1983, 55, 353-356.

34. Mann, M.; Aebersold, R. Mass spectrometry-based proteomics. Nature 2003, 422, 198-207. 